\title{
Molecular biomarker testing for non-small cell lung cancer: consensus statement of the Korean Cardiopulmonary Pathology Study Group
}

\author{
Sunhee Chang ${ }^{1}$, Hyo Sup Shim ${ }^{2}$, Tae Jung Kim ${ }^{3}$, Yoon-La Choi ${ }^{4}$, Wan Seop Kim ${ }^{5}$, Dong Hoon Shin ${ }^{6}$, Lucia Kim ${ }^{7}$, \\ Heae Surng Park ${ }^{8}$, Geon Kook Lee ${ }^{9}$, Chang Hun Lee ${ }^{10}$, Korean Cardiopulmonary Pathology Study Group \\ 1Department of Pathology, Inje University Ilsan Paik Hospital, Goyang; \\ 2Department of Pathology, Yonsei University College of Medicine, Seoul; \\ ${ }^{3}$ Department of Hospital Pathology, College of Medicine, The Catholic University of Korea, Seoul; \\ ${ }^{4}$ Department of Pathology, Samsung Medical Center, Sungkyunkwan University School of Medicine, Seoul; \\ ${ }^{5}$ Department of Pathology, Konkuk University School of Medicine, Seoul; \\ ${ }^{6}$ Department of Pathology, Pusan National University Yangsan Hospital, Yangsan; \\ ${ }^{7}$ Department of Pathology, Inha University School of Medicine, Incheon; \\ ${ }^{8}$ Department of Pathology, Ewha Womans University Seoul Hospital, Seoul; \\ ${ }^{9}$ Department of Pathology, National Cancer Center, Goyang; \\ ${ }^{10}$ Department of Pathology, Pusan National University Hospital, Pusan National University School of Medicine, Busan, Korea
}

\begin{abstract}
Molecular biomarker testing is the standard of care for non-small cell lung cancer (NSCLC) patients. In 2017, the Korean Cardiopulmonary Pathology Study Group and the Korean Molecular Pathology Study Group co-published a molecular testing guideline which contained almost all known genetic changes that aid in treatment decisions or predict prognosis in patients with NSCLC. Since then there have been significant changes in targeted therapies as well as molecular testing including newly approved targeted drugs and liquid biopsy. In order to reflect these changes, the Korean Cardiopulmonary Pathology Study Group developed a consensus statement on molecular biomarker testing. This consensus statement was crafted to provide guidance on what genes should be tested, as well as methodology, samples, patient selection, reporting and quality control.
\end{abstract}

Key Words: Carcinoma, non-small-cell lung; Biomarker; Precision medicine; Consensus

Received: October 30, 2020 Revised: March 18, 2021 Accepted: March 23, 2021

Corresponding Author: Chang Hun Lee, MD, Department of Pathology, Pusan National University Hospital, Pusan National University School of Medicine, 179 Gudeok-ro, Seo-gu, Busan 49241, Korea

Tel: +82-51-240-7718, Fax: +82-51-242-7422, E-mail: cnlee@pusan.ac.kr

Since the IRESSA Pan-Asia Study demonstrated that epidermal growth factor receptor $(E G F R)$ mutations are a predictive biomarker for response to EGFR tyrosine kinase inhibitor (TKI) therapy in non-small cell lung cancer (NSCLC) patients, biomarker testing has become the standard of care for NSCLC patients [1]. Currently, various targeted drugs and their predictive biomarkers have been approved by the Korea Ministry of Food and Drug Safety (MFDS) and U.S. Food and Drug Administration (FDA) (Table 1).

The Korean Cardiopulmonary Pathology Study Group (KCPSG) has developed molecular pathology guidelines to respond to these challenges [2-5]. In 2017, the KCPSG and the
Korean Molecular Pathology Study Group (KMPSG) jointly published a guideline for molecular testing, including almost all known genetic changes that aid in treatment decisions or predict prognosis in patients with NSCLC [4]. Since then, major changes have been made to targeted therapies and molecular tests, such as newly approved targeted drugs or liquid biopsies. In order to reflect recent changes, the KCPSG has crafted a consensus statement to address issues of which genes should be tested, methodology, samples, patient selection, reporting and quality control.

The purpose of this consensus statement is to provide standardized guidelines for molecular biomarker testing to help se- 
Table 1. Consensus statement of the Korean Cardiopulmonary Pathology Study Group

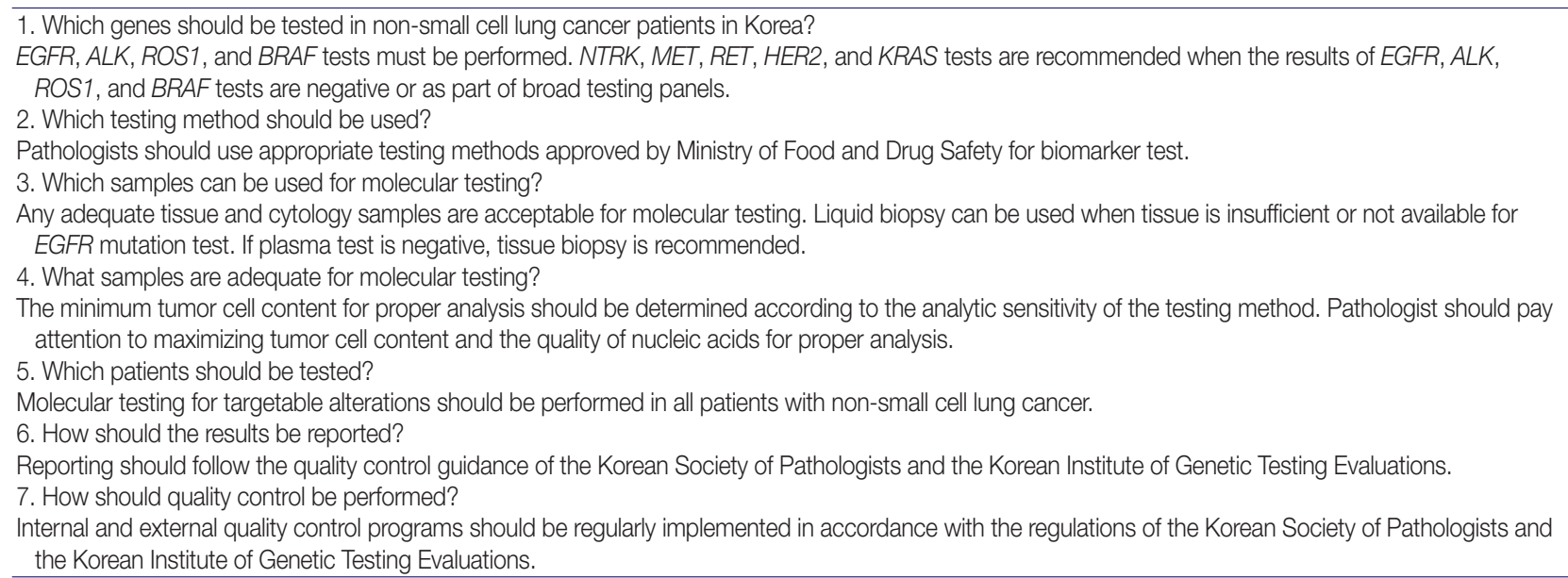

EGFR, epidermal growth factor receptor; ALK, anaplastic lymphoma kinase; ROS1, ros proto-oncogene 1 receptor tyrosine kinase; BRAF, serine/threonineprotein kinase B-raf; NTRK, neurotrophic tyrosine receptor kinase; MET, mesenchymal epithelial transition; RET, rearranged during transfection; HER2, human epidermal growth factor receptor 2; KRAS, kirsten rat sarcoma virus.

lect NSCLC patients for targeted therapy in Korea. The KCPSG Molecular Testing Working Group reviewed and assessed existing guidelines developed by the KCPSG/KMPSG [4], the College of American Pathologists (CAP)/International Association for the Study of Lung Cancer (IASLC)/Association for Molecular Pathology (AMP) [6], the American Society of Clinical Oncology (ASCO) [7], and the National Comprehensive Cancer Network (NCCN) [8]. The workgroup endorsed most recommendations within the existing guidelines, but made minor modifications based on recent changes in targeted therapy as well as current Korean medical system (Table 1).

\section{WHICH GENES SHOULD BE TESTED IN NON-SMALL CELL LUNG CANCER PATIENTS IN KOREA?}

\author{
EGFR, $A L K, R O S 1$, and BRAF tests must be performed; \\ NTRK, MET, RET, HER2, and KRAS tests are recommended \\ when the results of EGFR, ALK, ROS1, and BRAF tests are \\ negative or as part of broad testing panels
}

The important oncogenic drivers in NSCLC are mutations of $E G F R$, anaplastic lymphoma kinase $(A L K)$, ros proto-oncogene 1 receptor tyrosine kinase (ROS1), serine/threonine-protein kinase B-raf (BRAF), neurotrophic tyrosine receptor kinase (NTRK), mesenchymal epithelial transition (MET), rearranged during transfection (RET), human epidermal growth factor receptor 2 (HER2), and kirsten rat sarcoma virus (KRAS) [9]. Of these, sensitizing EGFR mutations, $A L K$ and ROS1 fusions, BRAF V600E mutations, NTRK fusions, MET exon 14 skipping mu- tations, and RET mutations or fusions, all have targeted drugs approved by the MFDS or FDA in NSCLC (Table 2). Targeted drugs for HER2 mutations are currently being investigated in clinical trials [10].

Sensitizing EGFR mutations occur within the kinase domain in exons 18 to 21. In particular, exon 19 deletions and exon 21 substitutions such as L858R account for about $90 \%$ of EGFR sensitizing mutations [9]. EGFR mutations occur in $37 \%$ to $40 \%$ of adenocarcinoma [11-13] and $9 \%$ of squamous cell carcinoma in Korea [13]. T790M is the most common resistance mechanism to EGFR TKI and occurs in 43\%-50\% of patients with acquired resistance to gefitinib/erlotinib [14]. Third-generation EGFR TKIs such as osimertinib inhibit both T790M and EGFR sensitizing mutations. Afatinib, erlotinib, gefitinib, and osimertinib have received reimbursement-approval by MFDS and National Health Insurance Service (NHIS) for NSCLC patients with EGFR mutations.

$A L K$ fusions occur in about $5 \%$ of NSCLC patients, primarily in young never-smokers with adenocarcinoma [3,9,15]. More than 20 different $A L K$ fusion partners have been discovered, of which EML4-ALK is the most common fusion protein in NSCLC $[3,9,15]$. Alectinib, brigatinib, ceritinib, and crizotinib have received reimbursement-approval for NSCLC patients with $A L K$ fusions.

ROS1 fusions are reported in 1\%-2\% of NSCLC patients, primarily in young never-smokers with adenocarcinoma [9]. ROS1 is related to the $A L K$ and insulin receptor superfamily. Because of high sequence homology to $A L K$, several ALK TKIs harbor dual inhibitory activity against $A L K$ and $R O S 1$ [9]. Crizo- 
Table 2. Targetable genetic alterations in non-small cell lung cancer patients

\begin{tabular}{|c|c|c|c|}
\hline Gene & Alteration & Method & Approved drug \\
\hline$\overline{E G F R}$ & $\begin{array}{l}\text { Mutation (Ex21L858R, } \\
\text { Ex19del, Ex18, Ex20) }\end{array}$ & $\begin{array}{l}\text { RT-PCR, NGS } \\
\text { (approved commercial test: PANAMutyper R EGFRa, Cobas EGFR Mutation } \\
\text { Test v2ª, GenesWell ddEGFR Mutation Test, Oncomine Dx Target Test }{ }^{b} \text { ) }\end{array}$ & Afatinib, erlotinib, gefitinib, osimertinib \\
\hline$A L K$ & Fusion & $\begin{array}{l}\text { Immunohistochemistry, FISH, NGS (approved commercial test: Vysis ALK Break } \\
\text { Apart FISH, ALK D5F3 CDx) }\end{array}$ & Alectinib, brigatinib, ceritinib, crizotinib \\
\hline ROS1 & Fusion & $\begin{array}{l}\text { Immunohistochemistryc, FISH, RT-PCR, NGS (approved commercial test: } \\
\text { AmoyDx ROS1 Gene Fusions Detection Kit, ROS1 SP384 Assayc, } \\
\text { Oncomine Dx Target Test }{ }^{b} \text { ) }\end{array}$ & Crizotinib \\
\hline BRAF & V600E mutation & $\begin{array}{l}\text { RT-PCR, NGS (approved commercial test: PNAClamp BRAF Mutation } \\
\text { Detection kitt } \text {, Oncomine Dx Target Test }{ }^{b} \text { ) }\end{array}$ & Dabrafenib+trametinib \\
\hline NTRK & Fusion & $\begin{array}{l}\text { Immunohistochemistryc, NGS } \\
\text { (approved commercial test: pan-TRK EPR17341 Assay }{ }^{\circ} \text { ) }\end{array}$ & Larotrectinib $^{d}$, Entrectinib ${ }^{d}$ \\
\hline MET & Exon 14 skipping mutation & NGS & Crizotinibe capmatinibib tepotinib $^{\dagger}$ \\
\hline RET & Fusion, mutations & NGS & Selpercatinibf \\
\hline HER2 & Mutation (Ex20ins) & NGS & Trastuzumabe \\
\hline TMB & & NGS (approved commercial test: FoundationOne CDx') & Pembrolizumab ${ }^{f}$ \\
\hline
\end{tabular}

EGFR, epidermal growth factor receptor; RT-PCR, real time polymerase chain reaction; NGS, next generation sequencing; ALK, anaplastic lymphoma kinase; FISH, fluorescent in situ hybridization; ROS1, ros proto-oncogene 1 receptor tyrosine kinase; BRAF, serine/threonine-protein kinase B-raf; NTRK, neurotrophic tyrosine receptor kinase; MET, mesenchymal epithelial transition; RET, rearranged during transfection; HER2, human epidermal growth factor receptor 2.

${ }^{a}$ Approved for tissue and plasma; ${ }^{b}$ Approved as new health technology; ' $F$ For screening; ${ }^{\circledR}$ Non-reimbursement approval; ${ }^{\mathrm{e}}$ Approval of non-reimbursement use of drugs exceeding the scope of product approval; 'Not approved in Korea (as of March 17, 2021).

tinib has received reimbursement-approval for NSCLC patients with ROS1 fusions.

$B R A F$ is a serine/threonine kinase downstream of KRAS in the mitogen-activated protein kinase (MAPK) signaling pathway. BRAF mutations occur in $1 \%-2 \%$ of NSCLC patients and are mostly represented by V600E [9]. Specific clinical or pathological features associated with BRAF mutations have not defined. Compared to BRAF inhibitor monotherapy, treatment with combination BRAF and MEK inhibitors improved response rates and progression free survival due to delays in MAPK-driven acquired resistance as well as reduced toxicities from paradoxical MAPK pathway activation $[8,9]$. The combination therapy with BRAF and MEK inhibitors has received reimbursementapproval for NSCLC patients with BRAF V600E mutations.

NTRK encodes for three transmembrane proteins, TRKA, TRKB, and TRKC, which play an important role in central nervous system development and maturation $[8,9]$. NTRK fusions have been identified in several types of solid tumors and occur in less than $1 \%$ of NSCLC patients $[8,9,16]$. Larotrectinib received non-reimbursement-approved for the NTRK-positive solid tumors including NSCLC.

Genetic alterations in MET such as exon 14 skipping mutations, amplification, and protein overexpression, are functionally important in cell proliferation, migration, and invasion $[8,9]$. MET exon 14 skipping mutations occur in $3 \%-4 \%$ of adenocarcinoma and $1 \%-2 \%$ of other NSCLC histologies [8,9]. TKIs targeting $M E T$ are subdivided into multikinase such as crizo- tinib and selective MET inhibitors such as tepotinib and capmatinib. Crizotinib, tepotinib, and capmatinib were granted breakthrough therapy status in 2018, 2019, and 2020, respectively, for the treatment of NSCLC patients with MET exon 14 skipping mutations by FDA. In Korea, crizotinib received approval for non-reimbursement use of drugs exceeding the scope of product approval.

RET fusions occur in $1 \%-2 \%$ of NSCLC and are more frequent in young never-smokers with adenocarcinomas [8,9]. Selpercatinib was granted breakthrough therapy status for the treatment of NSCLC patients with RET fusions by FDA in 2020. In Korea, ceritinib received approval for non-reimbursement use of drugs exceeding the scope of product approval.

HER 2 amplification and mutations are found in up to $4 \%$ and $35 \%$, respectively, of NSCLC [9]. Most HER2 mutations occur in exon 20 and lead to uncontrolled signaling activation through the same pathways activated by EGFR [9]. To date, no specific HER2 inhibitors have been approved and trials targeting HER2 exon 20 mutations or gene amplification have been investigated $[8,9]$. In Korea, trastuzumab received approval for non-reimbursement use of drugs exceeding the scope of product approval.

KRAS mutations occur in about $25 \%$ of NSCLC [8,9]. KRAS mutations are mutually exclusive to other oncogenic driver mutations and allow the identification of patients who are unlikely to have targetable alterations $[8,9]$. Studies aimed at exploring effective targeted therapies for KRAS mutations have been performed, with a recent clinical trial for KRAS G12C inhibitors 
showed promising results in preclinical and clinical settings [17]. The predictive role of KRAS mutations for response to chemotherapy, targeted therapy, anti-vascular therapy, or immunotherapy is currently controversial [17]. It is impractical to perform multiple single gene tests for all oncogenic drivers due to limited samples and as well as the financial burden on both patients and healthcare insurance. The 2018 CAP/IASLC/AMP guideline recommended that tests for EGFR, ALK, and ROS1 must be offered by all laboratories as an absolute minimum [6] BRAF, MET, RET, HER2, and KRAS should be included in any expanded panel if adequate material is available. All other genes can be considered for clinical trials at the time of publication. The 2018 ASCO guideline endorsed the CAP/IASLC/AMP guideline but added $B R A F$ in the absolute minimum category [7]. This is because dabrafenib/trametinib combination therapy for NSCLC with BRAF v600E mutations was approved in 2017 by FDA [6], just after the 2018 CAP/IASLC/AMP guideline panel completed its literature review $[7,18]$. The 2020 NCCN guideline also recommended tests for EGFR (category 1), $A L K$ (category 1), ROS1 (category 2A), and BRAF (category 2A) [8]. The 2020 NCCN guideline added tests for MET (category $2 \mathrm{~A}$ ) and $R E T$ (category $2 \mathrm{~A}$ ) based on recent data showing the efficacy and FDA approval of corresponding TKIs [8]. For HER2, KRAS, and NTRK, NCCN guideline strongly advised broad molecular profiling (category 2A) [8].

The extent of what constitutes the absolute minimum of predictive biomarkers should be decided by the approval and reimbursement status of the corresponding targeted therapies in each country. In Korea, targeted drugs for NSCLC patients with EGFR mutations, $A L K$ fusions, ROS1 fusions, or BRAF V600E mutations received reimbursement-approval as of October 15, 2020 (Table 1). Thus, we recommend EGFR, ALK, ROS1, and BRAF tests must be performed for treatment decision in NSCLC patients. Targeting drug for $M E T$ exon 14 skipping mutations, RET fusions, and HER2 mutations can be used only as non-reimbursement use of drugs exceeding the scope of product approval in NSCLC patients. NTRK TKI is approved as non-reimbursement. Therefore MET, RET, HER2, KRAS, and NTRK tests are recommended when the results of $E G F R, A L K, R O S 1$, and $B R A F$ tests are negative or as part of broad panels. Molecular testing for other genetic alterations can be performed for clinical trials.

Although not an oncogenic driver, tumor mutational burden (TMB) is an emerging biomarker for selection of patients with NSCLC for immunotherapy. TMB is defined as the number of mutations per megabase $(\mathrm{Mb})$ of DNA $[6,8,19]$. In NSCLC, smoking exposure and the associated genomic profile contribute significantly to high TMB [20]. Mutant proteins derived from somatic mutations produce neoantigens. Tumors with more neoantigens could elicit a stronger CD8 T-cell response in the presence of immunotherapy agents [20]. Therefore, TMB is considered a surrogate marker of neoantigen burden and therefore a predictive biomarker for immunotherapy. Several clinical trials have investigated tissue or blood based TMB as a predictive biomarker for immunotherapy [19]. The KEYNOTE-158 trials showed a higher response rate for patients with high TMB, which was defined as $\geq 10$ mutations/ $\mathrm{Mb}$ on a FoundationOne $\mathrm{CDx}$ assay (Foundation Medicine, Cambridge, MA, USA) to pembrolizumab [19,21].

\section{WHICH TESTING METHOD SHOULD BE USED?}

\section{Pathologists should use appropriate testing methods approved by MFDS for biomarker test}

It is important to consider the clinical utility, status of approval, and reimbursement in determining testing methods (Table 1). For EGFR testing, the CAP/ASCO/IASLC guideline recommended that testing methods must be able to detect molecular alterations in specimens with as little as $20 \%$ cancer cells. Furthermore, assays capable of detecting abnormality in as little as 5\% tumor cells should be used for EGFR T790M mutation [6]. Recently developed polymerase chain reaction (PCR)-based methods such as real time PCR (RT-PCR) and digital PCR (dPCR) are more sensitive than Sanger sequencing and can reliably detect low frequency mutations in samples with as little as 5\%-10\% cancer cells. In Korea, peptide nucleic acid clamping RT-PCR (Panagene, Daejeon, Korea), Cobas EGFR Mutation test (Roche, Indianapolis, IN, USA), pyrosequencing, and next generation sequencing (NGS) are commonly used for EGFR mutation test. Recently, droplet digital PCR (ddEGFR mutation test, Gencurix Inc., Seoul, Korea) and targeted NGS panel (Thermo Fisher Scientific, Waltham, MA, USA) have been approved as new health technologies. Mutation specific immunohistochemistry (IHC) is not encouraged for detection of EGFR mutations because it has low sensitivity and recent advances in molecular technology enable the detection of mutations in limited amounts of sample $[6,8]$. EGFR gene amplification or total protein expression should not be used to select patients for EGFR TKIs [6].

For $A L K$ fusions, fluorescent in situ hybridization (FISH) and IHC with ALK D5F3 CDx assay (Ventana Medical Systems Inc., Tucson, AZ, USA) are recommended [6,8,22]. Only Vysis ALK Break Apart FISH (Abbott Molecular Inc., Abbott Park, 
IL, USA) received approval for selection of NSCLC patients for treatment with brigatinib. The ALK D5F3 CDx assay has an overall sensitivity of $81 \%-100 \%$ and specificity of $91 \%-100 \%$. The ALK CDx is an equivalent alternative to ALK FISH $[6,8,22]$. The ALK CDx assay is widely used due to low cost, shorter turnaround time, and ease of use. It is important to understand several potential pitfalls of ALK CDx interpretation [22]. False positive staining, often weaker than true positive expression, may be seen in alveolar macrophages, airway epithelial cells, extracellular mucin, and necrotic debris. Tumor cells with neuroendocrine differentiation may show false positive staining although their expressions are typically heterogeneous or in a checkerboard pattern [22]. Therefore, samples with focal or equivocal expression are recommended to be retested with ALK FISH.

For ROS1 fusions, AmoyDx ROS1 gene fusions detection kit (Amoy Diagnostics Co., Xiamen, China) received approval for selection of NSCLC patients for crizotinib. FISH and NGS are available for detection of ROS1 fusion but cannot be used for treatment decisions in Korea. ROS1 IHC may be used as a screening test [8]. For BRAF V600E mutations, NGS and PNAClamp BRAF mutation detection kit (Panagene) can be used for treatment decision.

For other genetic alterations, the CAP/ASCI/IASLC guideline and NCCN guideline recommends that broad panels are preferred over multiple single gene tests to identify other treatment options $[6,8]$. We agree with this, and recommend NGS for NTRK, MET, RET, HER2, and KRAS. When NGS is not available, IHC may be used as a screening test for NTRK fusions and positive results should be confirmed by NGS [23]. NGS allows the assessment of many targetable genetic alterations from small samples at once $[8,24]$. Targeted NGS using amplicon resequencing enables the detection of point mutations with much higher sensitivity compared to single gene targeted tests $[8,24]$. NGS can detect gene fusions, in particular using an RNA-based approach [25]. Despite these advantages, NGS has not yet become a standard practice in Korea. NGS receives reimbursement only when performed by an approved medical institution. NGS is still more expensive than the sum of single gene tests for $E G F R$, $A L K, R O S 1$, and $B R A F$ as of 2020 in Korea. In addition, NGS is not approved for treatment decisions for NSCLC patients with ROS1 or ALK fusions. A detailed laboratory guideline for NGS is beyond the scope of this paper (see Kim et al. [26] for guidelines and requirements for clinical NGS tests).

\section{WHICH SAMPLES CAN BE USED FOR MOLECULAR TESTING?}

Any adequate tissue and cytology samples are acceptable for molecular testing; Liquid biopsy can be used when tissue is insufficient or not available for EGFR mutation test; If plasma test is negative, tissue biopsy is recommended

The CAP, ASCO, and NCCN guidelines recommended that any tissue and cytology samples with adequate cellularity and preservation are suitable for molecular testing [6-8]. We agree this recommendation and encourage active utilization of cytology samples for molecular testing because patients with advanced lung cancer often cannot undergo tissue biopsy or, may have biopsies with insufficient tumor tissue.

Various cytology preparations including cell blocks, direct smears, cytospin preparations, and liquid-based cytology are acceptable and reliable for biomarker testing [6-8]. Molecular testing results using cytology samples are highly concordant with those of the corresponding tissue samples, in particular with more sensitive methods [27-32]. A study comparing concurrently acquired fine needle aspiration and core needle biopsy samples showed better cellularity, higher tumor fraction, and higher mutation allelic frequencies in aspiration smears than biopsy samples [30]. It is important to understand the advantages and disadvantages of cytology samples for appropriate triaging of biomarker tests.

Formalin-fixed, paraffin-embedded (FFPE) cell blocks are widely used for molecular testing. The main advantage of cell blocks is to allow serial sections for multiple tests. Because most biomarker assays have been validated on FFPE samples, cell blocks do not require additional validation [33-38]. Compared to alcohol-fixed cytology samples, the limitations of cell blocks are due to formalin artifacts in DNA and nuclear truncation [33-36]. Formalin leads to cross-linking and chemical modification of nucleic acids which may affect DNA quality. In FISH, the presence of truncated nuclei in cellblock sections may result in artifactual loss of probe signal, as seen in conventional FFPE block [3]. Cell blocks often exhibit depletion of tumor cells on deeper sections.

Air dried or alcohol-fixed cytology samples such as smears and cytospins may offer higher quality nucleic acids than cell blocks [33-38]. Smears, cytospins, and liquid-based cytology allow an evaluation of the adequacy and cellularity of tumor cells on site and the opportunity to scrape tumor cells by macro/microdissection [33-38]. They enable the presentation of whole tumor nuclei for FISH [3]. The main limitation of smears is the sacri- 
fice of archival stained slides. Whole slide imaging may be used for archiving cytology slides. The use of non-FFPE cytology samples may require further validation of molecular testing. ALK CDx has not been approved for non-FFPE cytology samples.

Tissue-based molecular testing is often not available in NSCLC patients due to the invasiveness and high risk of complications of biopsy procedures or due to insufficient tumor tissue obtained [5,39]. In addition, failure rate for re-biopsy was reported to be about 20\% in NSCLC patients with progression or metastasis [40]. The liquid biopsy is a non-invasive way to detect genetic alterations. Tumor biopsies are often not able to encompass comprehensive genomic profiles due to tumor heterogeneity. However liquid biopsy is more likely representative of whole tumor clones because tumor DNA is constantly released into the bloodstream from all tumor sites [39,41]. Studies using various analytes such as circulating tumor cells, circulating tumor DNA (ctDNA), tumor educated platelets and tumor-derived exosomes obtained from blood or other body fluids have shown promising results for detection of genetic alterations in NSCLC patients. But, plasma ctDNA assay is only approved by MFDS and FDA. Plasma ctDNA testing for detection of $E G F R$ mutations to select patients for EGFR TKIs has been covered in Korea since 2018.

The limitation of liquid biopsy is that ctDNA level is often extremely low and requires highly sensitive techniques. Moreover, germline cell free DNA (cfDNA) contamination from white cells could dilute ctDNA levels. In meta-analysis studies, the sensitivity of plasma ctDNA assay was about $67 \%$ [6,42]. The sensitivity can be increased up to $72 \%$ and $87 \%$ by use of more sensitive methods such as $\mathrm{dPCR}$ and NGS, respectively [42]. The specificity of plasma ctDNA assay was $96 \%$ for overall mutations and $80 \%$ for $\mathrm{T} 790 \mathrm{M}[6,42]$. The relatively low specificity could be in part, due to the high tumor heterogeneity, which can lead to false negative results in tissue tests [42]. This is supported by results of clinical trials showing that plasma T790Mpositive patients had similar outcomes to tissue T790M-positive patients for osimertinib treatment $[43,44]$. Therefore, plasma testing should be performed in patients whose tissue material is unavailable or insufficient. And if plasma test is negative, repeat biopsy should be done for EGFR testing and other genetic alterations.

\section{WHAT SAMPLES ARE ADEQUATE FOR MOLECULAR TESTING?}

The minimum tumor cell content for proper analysis should be determined according to the analytic sensitivity of the testing method. Pathologist should pay attention to maximizing tumor cell content and the quality of nucleic acids for proper analysis

Sample adequacy is determined by the quantity and quality of tumor cells. The minimum tumor cell content for mutational testing is largely dependent on the analytic sensitivity of the testing method. Recently developed RT-PCR and dPCR techniques require samples with a minimum of $10 \%$ tumor cell content. However, in an unpublished survey of KCPSG, half of respondents said they performed $E G F R$ tests even when tumor cell content was less than $10 \%$ or there were less than 200 tumor cells. Various approaches for tumor saving and enrichment enable the detection of genetic alterations even in samples with a tumor content less than the platform threshold [45].

The most commonly used strategy for tumor enrichment is manual macro/microdissection [45]. Pathologists review hematoxylin and eosin $(\mathrm{H} \& \mathrm{E})$ stained slides and mark tumor areas directly on the slide. Subsequently, the corresponding areas are manually scraped off from the slides or procured directly from the block using the marked $\mathrm{H} \& \mathrm{E}$ slide as a guide. The EURTAC trial showed similar analytic accuracy in samples with less than or equal to $10 \%$ tumor content with microdissection, compared to samples with more than $10 \%$ without microdissection for EGFR test [46]. Samples with more than or equal to 30\% tumor content in the dissected area showed good success rates of $95.6 \%$, regardless of the size of the dissected area [31].

In small samples such as needle biopsy or cell block, tumor cell content is often limited and disappears on deeper sections. Thus, tumor saving is important for biomarker testing. The most effective tumor saving strategy is the pathologist ordered reflex test, in which the pathologist orders a predefined set of biomarker tests at the time of pathologic diagnosis [45,47]. In reflex testing, predefined tests are performed all at once using pre-cut unstained slides. This avoids tissue waste due to trimming or refacing and increases the quality of molecular testing [45,47-49]. In addition, this standardized and comprehensive approach by the pathologist led to reductions in turnaround time and an increase in the number of patients with biomarker testing results available at the time of treatment decision $[48,49]$. The limitation of reflex testing is that early-stage NSCLCs would be included. Several trials for adjuvant and neoadjuvant target therapy in early-stage NSCLC 
patients are ongoing with promising preliminary results [50,51]. The aura 3 trial showed longer disease-free survival among those who received osimertinib than among those who received placebo in patients with stage II to IIIA EGFR mutation-positive NSCLC [51].

The second strategy for tissue saving is to make one block for one core for needle biopsy specimen. The one core-one block strategy also avoids unnecessary trimming and allows representative cross-sections of all tissue cores embedded in a single block [45]. Multiple blocks are distributed to appropriate tests according to the tumor cell content of individual blocks, allowing efficient use of the tissue [45]. Finally, IHC to distinguish between adenocarcinoma and squamous cell carcinoma should be minimized in small samples. Large IHC panels may not provide an advantage over a limited immunohistochemical workup using thyroid transcription factor 1 (or Napsin A) and p40 (or p63) [8,52].

ALK or ROS1 FISH requires a minimum of 50 to 100 viable tumor cells based on the interpretation guideline and if the result is equivocal, an additional 50 nuclei should be evaluated by the second reader [4]. It is recommended to avoid areas of necrosis and areas where the nuclear borders overlap or are indistinguishable from the adjacent stromal cells. If the tumor is very focal within the sample, the area to be examined on the slide can be marked for easy identification with a dark field fluorescence microscope. The ALK D5F3 CDx can be performed on FFPE samples with any tumor cells.

The important preanalytical factors affecting nucleic acid and protein quality are cold ischemia time, total fixation time, fixatives, and decalcification $[53,54]$. One hour or less of cold ischemia time (time between specimen removal from the body and its stabilization in formalin) is recommended [53,54]. Cold ischemia time of less than 1 hour for FISH, less than 2 hours for protein and RNA, and 24 hours or less for DNA have been reported to be acceptable [53]. The recommended fixative is $10 \%$ neutral, phosphate-buffered formalin. The $10 \%$ neutral buffered formalin is the most commonly used fixative for routine histology, providing excellent morphological preservation. Most IHC and molecular tests are validated for FFPE samples. Total fixation time of 6 to 24 hours is recommended. Fixation time of 6 to 24 hours for protein, 8 to 48 hours for RNA, and less than 72 hours for DNA is acceptable [53,54]. Strict quality control for concentration and $\mathrm{pH}$ of formalin and total fixation time is required to minimize formalin artifacts that can affect nuclei acid [54]. Of liquid based cytology preservative, CytoRich Red containing small amounts of formaldehyde may affect DNA yield [55]. Ethylenediaminetetraacetic acid (EDTA) is recom- mended for decalcification, in particular for needle biopsy and small samples $[56,57]$. EDTA preserves morphology and nucleic acid but slowly decalcifies. Decalcifying agents containing strong acids do not yield adequate DNA for molecular testing but rapidly decalcify. Use of hydrochloric acid should be limited to large resection specimens such as rib and the ability to be cut with a blade should be monitored every day.

The ctDNA is rapidly degraded by nucleases and contaminated by non-tumor DNA from white cells in whole blood [5]. Therefore, plasma should be separated as quickly as possible after the blood draw. Specialized cfDNA stabilizing tubes can be used for longer storage times. For future analysis, plasma should be frozen in single use fractions until DNA extraction [54].

\section{WHICH PATIENTS SHOULD BE TESTED?}

\section{Molecular testing for targetable alterations should be performed in all patients with non-small cell lung cancer}

It is uncontroversial that molecular testing for targetable alterations should be performed in all patients with adenocarcinoma or an adenocarcinoma component [6-8]. For tumors with other histologies, the CAP guideline recommends that biomarker testing may be performed when clinical features, such as young age and never-smoker, indicate a higher probability of targetable alterations [6]. The ASCO guideline recommends biomarker testing for patients with nonsquamous NSCLC or with squamous cell carcinoma who are light or never-smokers or younger than 50 years of age [7]. The NCCN guideline recommends biomarker testing for patients with large cell carcinoma or NSCLC not otherwise specified (category 1) [8]. For squamous cell carcinoma, tests for EGFR and ALK should be considered in never-smokers or small biopsy specimens, or those with mixed histology (category 2A) and tests for ROS1, BRAF, MET, and RET should be considered in small biopsies or mixed histologies (category 2A) [8].

In squamous cell carcinoma, the frequency of EGFR mutations is about $7 \%-8 \%$ and $A L K$ fusion occurs in less than $1 \%$ $[13,58,59]$. The detection of EGFR mutations in small samples diagnosed as squamous cell carcinoma may be a result of incomplete sampling of adenosquamous cell carcinoma [59-62]. Because of histologic heterogeneity, small biopsies and cytology samples may not be representative of the total tumor and an adenocarcinoma component cannot be completely excluded. Undersampling of adenosquamous cell carcinoma is possible even in resection specimens of which only representative sections are submitted for pathologic examination. Several studies have 
shown that the adenocarcinoma component and squamous cell carcinoma component of most adenosquamous cell carcinomas shared genetic alterations including EGFR mutations [60,63]. Restriction to adenocarcinoma histology may exclude some patients with squamous cell carcinoma from the potential benefits of targeted therapy. Therefore, molecular testing for targetable alterations should be performed in all NSCLC to select patients for targeted therapy.

\section{HOW SHOULD THE RESULTS BE REPORTED?}

Reporting should follow the quality control guidance of the Korean Society of Pathologists and the Korean Institute of Genetic Testing Evaluations

Reporting on molecular tests should follow the quality control guidance of the Korean Society of Pathologists (KSP) and the Korean Institute of Genetic Testing Evaluations (KIGTE). Reporting should provide information the clinician needs for arriving at treatment decisions. When reporting EGFR mutations, the clinical significance of the results should be included (e.g., response to EGFR TKI, resistance to EGFR TKI, or limited data on response). The sample adequacy and any limitations of the testing methods should be included for correct interpretation of the result by the clinician.

\section{HOW SHOULD QUALITY CONTROL BE PERFORMED?}

\section{Internal and external quality control programs should be} regularly implemented in accordance with the regulations of the Korean Society of Pathologists and the Korean Institute of Genetic Testing Evaluations

Regular quality control is necessary to maintain a high degree of reliability in molecular testing. The KSP and KIGTE provide guidance on internal quality control. Laboratories should also be enrolled in an external quality control and quality improvement program of the KSP and the KIGTE.

\section{FUTURE PERSPECTIVES}

With advances in precision medicine, NGS will become a routine practice. Tumor samples are often too limited to enable the detection of all targetable alterations with multiple single genetic tests. And biomarkers such as TMB can only be tested with NGS. Targeted NGS can rapidly and comprehensively detect multiple genetic alterations and save tumor tissue. The cost of
NGS is dropping rapidly. However, issues of reimbursement and approval with regard to NGS will need to be resolved for lung cancer patients to benefit from this technology. In USA, FoundationOne CDx, Oncomine Dx Target Test (Thermo Fisher Scientific, Carlsbad, CA, USA), FoundationOne Liquid CDx (Foundation Medicine), and Guardant360 CDx (Guardant Health, Inc., Redwood City, CA, USA) are currently FDA approved NGS panels as companion diagnostics for NSCLC patients. FoundationOne $\mathrm{CDx}$ detects genetic alterations in 324 genes and provides information on microsatellite instability and TMB using DNA isolated from FFPE tissue. Oncomine Dx Target Test detects genetic alterations in 23 genes using DNA and fusions in ROS1 using RNA from FFPE tissue. FoundationOne Liquid CDx and Guardant $360 \mathrm{CDx}$ detects on genetic alterations in 311 genes and 55 genes, respectively, using ctDNA from plasma. And these two panels have been utilized for evaluation of blood TMB in clinical trials [19].

TMB may be used as a biomarker for immunotherapy in the foreseeable future. Although the FDA has approved high TMB as a biomarker for pembrolizumab treatment of solid tumors, TMB is still considered an emerging biomarker for NSCLC patients in the NCCN guideline [8]. Different assays, platforms and cutoffs to assess TMB have been used in many clinical trials and retrospective analyses [64]. Therefore, standardization of TMB assessment is key to its use in clinical practice. The ctDNA analysis could be used as a monitoring tool for treatment responses as recent clinical trials have demonstrated a correlation between changes in, or clearance of plasma EGFR mutational burden with EGFR TKI response $[19,65]$. However, consensus on how to appropriately measure treatment response has not yet been established.

\section{CONCLUSION}

In the era of targeted therapies and personalized medicine, the role of the pathologists is important in the management of NSCLC patients. At a minimum, tests for EGFR, ALK, ROS1, and $B R A F$ should be performed for treatment decisions in all NSCLC patients. In the near future, NTRK, MET, RET, HER2, and $K R A S$ tests will also be mandatory tests. Although advances in technology allow the detection of molecular alterations not only in tissue and cytology samples but also in ctDNA, tumor sample limitations are still a major challenge for molecular testing. Pathologists should pay attention to tumor saving strategies and enrichment through standardized and comprehensive approaches. Pathologists must also utilize a regular quality control program for 
reliable analyses. New targeted therapies, predictive biomarkers, and technologies are continually emerging. KCPSG will keep pace with these advances through regular updates of these molecular guidelines for lung cancer patients.

\section{Ethics Statement}

Not applicable.

\section{Availability of Data and Material}

Data sharing not applicable to this article as no datasets were generated or analyzed during the study.

\section{Code Availability}

Not applicable.

\section{ORCID}

Sunhee Chang Hyo Sup Shim Tae Jung Kim Yoon-La Choi Wan Seop Kim Dong Hoon Shin Lucia Kim Heae Surng Park Geon Kook Lee Chang Hun Lee

\section{Author Contributions}

Conceptualization: GKL, YLC, SC. Data curation: SC. Formal analysis: SC. Investigation: SC, HSS, TJK, YLC, WSK, DHS, LK, HSP. Methodology: SC, HSS, TJK. Project administration: SC. Supervision: CHL, YLC. Writingoriginal draft preparation: SC. Writing—review \& editing: HSS, WSK, CHL. Approval of final manuscript: all authors.

\section{Conflicts of Interest}

The authors declare that they have no potential conflicts of interest.

\section{Funding Statement}

This research was supported by a 2-year research grant from Pusan National University.

\section{References}

1. Fukuoka M, Wu YL, Thongprasert S, et al. Biomarker analyses and final overall survival results from a phase III, randomized, open-label, first-line study of gefitinib versus carboplatin/paclitaxel in clinically selected patients with advanced non-small-cell lung cancer in Asia (IPASS). J Clin Oncol 2011; 29: 2866-74.

2. Shim HS, Chung JH, Kim L, et al. Guideline recommendations for EGFR mutation testing in lung cancer: proposal of the Korean Cardiopulmonary Pathology Study Group. Korean J Pathol 2013; 47: 100-6.

3. Kim H, Shim HS, Kim L, et al. Guideline recommendations for testing of $A L K$ gene rearrangement in lung cancer: a proposal of the Korean Cardiopulmonary Pathology Study Group. Korean J Pathol 2014; 48: 1-9.

4. Shim HS, Choi YL, Kim L, et al. Molecular testing of lung cancers. J Pathol Transl Med 2017; 51: 242-54.

5. Shin DH, Shim HS, Kim TJ, et al. Provisional guideline recommendation for EGFR gene mutation testing in liquid samples of lung cancer patients: a proposal by the Korean Cardiopulmonary Pathology Study Group. J Pathol Transl Med 2019; 53: 153-8.

6. Lindeman NI, Cagle PT, Aisner DL, et al. Updated molecular testing guideline for the selection of lung cancer patients for treatment with targeted tyrosine kinase inhibitors: guideline from the College of American Pathologists, the International Association for the Study of Lung Cancer, and the Association for Molecular Pathology. Arch Pathol Lab Med 2018; 142: 321-46.

7. Kalemkerian GP, Narula N, Kennedy EB, et al. Molecular testing guideline for the selection of patients with lung cancer for treatment with targeted tyrosine kinase inhibitors: American Society of Clinical Oncology Endorsement of the College of American Pathologists/International Association for the Study of Lung Cancer/ Association for Molecular Pathology clinical practice guideline update. J Clin Oncol 2018; 36: 911-9.

8. Ettinger DS, Wood DE, Aisner DL, et al. NCCN Clinical practice guidelines in oncology (NCCN Guidelines) for non-small cell lung cancer, version 8.2020 [Internet]. Plymouth Meeting: National Comprehensive Cancer Network, 2020 [cited 2020 Oct 15]. Available from: https://www.nccn.org/professionals/physician_gls/pdf/ nscl.pdf.

9. Genova C, Rossi G, Tagliamento M, et al. Targeted therapy of oncogenic-driven advanced non-small cell lung cancer: recent advances and new perspectives. Expert Rev Respir Med 2020; 14: 367-83.

10. Del Re M, Cucchiara F, Petrini I, et al. erbB in NSCLC as a molecular target: current evidences and future directions. ESMO Open 2020; 5 : e000724.

11. Yoon HY, Ryu JS, Sim YS, et al. Clinical significance of EGFR mutation types in lung adenocarcinoma: a multi-centre Korean study. PLoS One 2020; 15: e0228925.

12. Choi CM, Kim HC, Jung CY, et al. Report of the Korean Association of Lung Cancer Registry (KALC-R), 2014. Cancer Res Treat 2019; 51: 1400-10.

13. Lee SH, Kim WS, Choi YD, et al. Analysis of mutations in epidermal growth factor receptor gene in Korean patients with non-small cell lung cancer: summary of a nationwide survey. J Pathol Transl Med 2015; 49: 481-8.

14. Janne PA. Challenges of detecting EGFR T790M in gefitinib/erlotinib-resistant tumours. Lung Cancer 2008; 60 Suppl 2: S3-9.

15. Kwak EL, Bang YJ, Camidge DR, et al. Anaplastic lymphoma kinase inhibition in non-small-cell lung cancer. N Engl J Med 2010; 363: 1693-703.

16. Farago AF, Taylor MS, Doebele RC, et al. Clinicopathologic features of non-small-cell lung cancer harboring an NTRK gene fusion. JCO Precis Oncol 2018;2018:PO.18.00037.

17. Ghimessy A, Radeczky P, Laszlo V, et al. Current therapy of KRASmutant lung cancer. Cancer Metastasis Rev 2020; 39: 1159-77.

18. Pennell NA, Arcila ME, Gandara DR, West H. Biomarker testing for patients with advanced non-small cell lung cancer: real-world issues and tough choices. Am Soc Clin Oncol Educ Book 2019; 39: 531-42.

19. Rolfo C, Cardona AF, Cristofanilli M, et al. Challenges and opportunities of cfDNA analysis implementation in clinical practice: perspective of the International Society of Liquid Biopsy (ISLB). Crit Rev Oncol Hematol 2020; 151: 102978.

20. Tafe LJ. Non-small cell lung cancer as a precision oncology para- 
digm: emerging targets and tumor mutational burden (TMB). Adv Anat Pathol 2020; 27: 3-10.

21. Marabelle A, Fakih M, Lopez J, et al. Association of tumour mutational burden with outcomes in patients with advanced solid tumours treated with pembrolizumab: prospective biomarker analysis of the multicohort, open-label, phase 2 KEYNOTE-158 study. Lancet Oncol 2020; 21: 1353-65.

22. Uruga H, Mino-Kenudson M. ALK (D5F3) CDx: an immunohistochemistry assay to identify ALK-positive NSCLC patients. Pharmgenomics Pers Med 2018; 11: 147-55.

23. Marchio C, Scaltriti M, Ladanyi M, et al. ESMO recommendations on the standard methods to detect NTRK fusions in daily practice and clinical research. Ann Oncol 2019; 30: 1417-27.

24. Ku BM, Heo MH, Kim JH, et al. Molecular screening of small biopsy samples using next-generation sequencing in Korean patients with advanced non-small cell lung cancer: Korean Lung Cancer Consortium (KLCC-13-01). J Pathol Transl Med 2018; 52: 148-56.

25. Bruno R, Fontanini G. Next generation sequencing for gene fusion analysis in lung cancer: a literature review. Diagnostics (Basel) 2020; 10: 521.

26. Kim J, Park WY, Kim NK, et al. Good laboratory standards for clinical next-generation sequencing cancer panel tests. J Pathol Transl Med 2017; 51: 191-204.

27. Lee SE, Lee SY, Park HK, et al. Detection of EGFR and KRAS mutation by pyrosequencing analysis in cytologic samples of non-small cell lung cancer. J Korean Med Sci 2016; 31: 1224-30.

28. Sun PL, Jin Y, Kim H, Lee CT, Jheon S, Chung JH. High concordance of EGFR mutation status between histologic and corresponding cytologic specimens of lung adenocarcinomas. Cancer Cytopathol 2013; 121:311-9.

29. Asaka S, Yoshizawa A, Nakata R, et al. Utility of bronchial lavage fluids for epithelial growth factor receptor mutation assay in lung cancer patients: comparison between cell pellets, cell blocks and matching tissue specimens. Oncol Lett 2018; 15: 1469-74.

30. Roy-Chowdhuri S, Chen H, Singh RR, et al. Concurrent fine needle aspirations and core needle biopsies: a comparative study of substrates for next-generation sequencing in solid organ malignancies. Mod Pathol 2017; 30: 499-508.

31. Shiau CJ, Babwah JP, da Cunha Santos G, et al. Sample features associated with success rates in population-based EGFR mutation testing. J Thorac Oncol 2014; 9: 947-56.

32. da Cunha Santos G, Wyeth T, Reid A, et al. A proposal for cellularity assessment for EGFR mutational analysis with a correlation with DNA yield and evaluation of the number of sections obtained from cell blocks for immunohistochemistry in non-small cell lung carcinoma. J Clin Pathol 2016; 69: 607-11.

33. Roy-Chowdhuri S, Aisner DL, Allen TC, et al. Biomarker testing in lung carcinoma cytology specimens: a perspective from members of the Pulmonary Pathology Society. Arch Pathol Lab Med 2016; 140: $1267-72$

34. Lozano MD, Echeveste JI, Abengozar M, et al. Cytology smears in the era of molecular biomarkers in non-small cell lung cancer: doing more with less. Arch Pathol Lab Med 2018; 142: 291-8.

35. Jain D, Roy-Chowdhuri S. Molecular pathology of lung cancer cytology specimens: a concise review. Arch Pathol Lab Med 2018; 142: 1127-33.

36. da Cunha Santos G, Saieg MA, Troncone G, Zeppa P. Cytological preparations for molecular analysis: a review of technical proce- dures, advantages and limitations for referring samples for testing. Cytopathology 2018; 29: 125-32.

37. Auger M, Brimo F, Kanber Y, Fiset PO, Camilleri-Broet S. A practical guide for ancillary studies in pulmonary cytologic specimens. Cancer Cytopathol 2018; 126 Suppl 8: 599-614.

38. Aisner DL. The revised College of American Pathologists/International Association for the Study of Lung Cancer/Association for Molecular Pathology guideline: a step forward for molecular cytopathology. Arch Pathol Lab Med 2018; 142: 684-5.

39. Chang S, Hur JY, Choi YL, Lee CH, Kim WS. Current status and future perspectives of liquid biopsy in non-small cell lung cancer. J Pathol Transl Med 2020; 54: 204-12.

40. Nosaki K, Satouchi M, Kurata T, et al. Re-biopsy status among nonsmall cell lung cancer patients in Japan: a retrospective study. Lung Cancer 2016; 101: 1-8.

41. Siravegna G, Marsoni S, Siena S, Bardelli A. Integrating liquid biopsies into the management of cancer. Nat Rev Clin Oncol 2017; 14: 531-48.

42. Passiglia F, Rizzo S, Di Maio M, et al. The diagnostic accuracy of circulating tumor DNA for the detection of EGFR-T790M mutation in NSCLC: a systematic review and meta-analysis. Sci Rep 2018; 8: 13379.

43. Oxnard GR, Thress KS, Alden RS, et al. Association between plasma genotyping and outcomes of treatment with osimertinib (AZD9291) in advanced non-small-cell lung cancer. J Clin Oncol 2016; 34: 337582.

44. Remon J, Caramella C, Jovelet C, et al. Osimertinib benefit in EGFR-mutant NSCLC patients with T790M-mutation detected by circulating tumour DNA. Ann Oncol 2017; 28: 784-90.

45. Aisner DL, Rumery MD, Merrick DT, et al. Do more with less: tips and techniques for maximizing small biopsy and cytology specimens for molecular and ancillary testing: the University of Colorado Experience. Arch Pathol Lab Med 2016; 140: 1206-20.

46. Cobas EGFR mutation test v2 [Internet]. Silver Spring: U.S. Food and Drug Administration, 2020 [cited 2020 Oct 15]. Available from: https://www.accessdata.fda.gov/cdrh_docs/pdf12/P120019S007c. pdf.

47. Lim C, Sekhon HS, Cutz JC, et al. Improving molecular testing and personalized medicine in non-small-cell lung cancer in Ontario. Curr Oncol 2017; 24: 103-10.

48. Anand K, Phung TL, Bernicker EH, Cagle PT, Olsen RJ, Thomas JS. Clinical utility of reflex ordered testing for molecular biomarkers in lung adenocarcinoma. Clin Lung Cancer 2020; 21: 437-42.

49. Cheema PK, Menjak IB, Winterton-Perks Z, et al. Impact of reflex EGFR/ ALK testing on time to treatment of patients with advanced nonsquamous non-small-cell lung cancer. J Oncol Pract 2017; 13: e130-8.

50. Indini A, Rijavec E, Bareggi C, Grossi F. Novel treatment strategies for early-stage lung cancer: the oncologist's perspective. J Thorac Dis 2020; $12: 3390-8$.

51. Wu YL, Tsuboi M, He J, et al. Osimertinib in resected EGFR-mutated non-small-cell lung cancer. N Engl J Med 2020; 383: 1711-23.

52. Pelosi G, Fabbri A, Bianchi F, et al. DeltaNp63 (p40) and thyroid transcription factor-1 immunoreactivity on small biopsies or cellblocks for typing non-small cell lung cancer: a novel two-hit, sparingmaterial approach. J Thorac Oncol 2012; 7: 281-90.

53. Bass BP, Engel KB, Greytak SR, Moore HM. A review of preanalytical factors affecting molecular, protein, and morphological analysis 
of formalin-fixed, paraffin-embedded (FFPE) tissue: how well do you know your FFPE specimen? Arch Pathol Lab Med 2014; 138: 1520-30.

54. Compton CC, Robb JA, Anderson MW, et al. Preanalytics and precision pathology: pathology practices to ensure molecular integrity of cancer patient biospecimens for precision medicine. Arch Pathol Lab Med 2019; 143: 1346-63.

55. Dejmek A, Zendehrokh N, Tomaszewska M, Edsjo A. Preparation of DNA from cytological material: effects of fixation, staining, and mounting medium on DNA yield and quality. Cancer Cytopathol 2013; 121: 344-53.

56. Miquelestorena-Standley E, Jourdan ML, Collin C, et al. Effect of decalcification protocols on immunohistochemistry and molecular analyses of bone samples. Mod Pathol 2020; 33: 1505-17.

57. Choi SE, Hong SW, Yoon SO. Proposal of an appropriate decalcification method of bone marrow biopsy specimens in the era of expanding genetic molecular study. J Pathol Transl Med 2015; 49: 23642.

58. COSMIC (catalogue of somatic mutations in cancer) [Internet]. COSMIC, 2020 [cited 2020 Oct 15]. Available from: https://cancer. sanger.ac.uk/cosmic.

59. Wang H, Sun L, Sang Y, et al. A study of ALK-positive pulmonary squamous-cell carcinoma: From diagnostic methodologies to clinical efficacy. Lung Cancer 2019; 130: 135-42.
60. Paik PK, Varghese AM, Sima CS, et al. Response to erlotinib in patients with EGFR mutant advanced non-small cell lung cancers with a squamous or squamous-like component. Mol Cancer Ther 2012; 11: $2535-40$

61. Dragnev KH, Gehr G, Memoli VA, Tafe LJ. ALK-rearranged adenosquamous lung cancer presenting as squamous cell carcinoma: a potential challenge to histologic type triaging of NSCLC biopsies for molecular studies. Clin Lung Cancer 2014; 15: e37-40.

62. Mamesaya N, Nakashima K, Naito T, Nakajima T, Endo M, Takahashi T. $A L K$-rearranged lung squamous cell carcinoma responding to alectinib: a case report and review of the literature. BMC Cancer 2017; 17: 471.

63. Lin G, Li C, Li PS, et al. Genomic origin and EGFR-TKI treatments of pulmonary adenosquamous carcinoma. Ann Oncol 2020; 31: 517-24.

64. Sholl LM, Hirsch FR, Hwang D, et al. The promises and challenges of tumor mutation burden as an immunotherapy biomarker: a perspective from the International Association for the Study of Lung Cancer Pathology Committee. J Thorac Oncol 2020; 15: 1409-24.

65. Sakai K, Takahama T, Shimokawa M, et al. Predicting osimertinibtreatment outcomes through EGFR mutant-fraction monitoring in the circulating tumor DNA of EGFR T790M-positive patients with non-small cell lung cancer (WJOG8815L). Mol Oncol 2021; 15: 126-37. 